\title{
A New Set of Normalized Geometric Moments Based on Schlick's Approximation
}

\author{
Ramakrishnan Mukundan \\ Department of Computer Science and Software Engineering \\ University of Canterbury, Christchurch, New Zealand
}

\begin{abstract}
Schlick's approximation of the term $x^{p}$ is used primarily to reduce the complexity of specular lighting calculations in graphics applications. Since moment functions have a kernel defined using a monomial $x^{p} y^{p}$, the same approximation could be effectively used in the computation of normalized geometric moments and invariants. This paper outlines a framework for computing moments of various orders of an image using a simplified kernel, and shows the advantages provided by the approximating function through a series of experimental results.
\end{abstract}

\section{Introduction}

Geometric moments and moment invariants have been extensively used as feature descriptors in several pattern recognition and vision related applications [1],[2],[3]. Even though many other types of complex and orthogonal moments have also been developed in order to get improved feature representation capability in a moment set, geometric moments continue to be popular as they have a simple structure that can be easily implemented. A number of fast algorithms for the computation of geometric moments can therefore be found in literature [4],[5], including VLSI implementations [6]. Geometric moments are usually normalized or scaled to reduce the dynamic range of values. Most commonly found applications of geometric moments are pattern recognition, template matching, image classification, and pose estimation.

This paper proposes a completely new approach to computation of normalized geometric moments and their invariants using Schlick's approximation. The approximation of the term $x^{p}$ by a simple fraction of polynomial functions when $x$ has a value less than 1 , is found to be useful in lighting calculations involving specular reflections[7],[8]. Lighting involves a huge amount of computations in many graphics applications with large polygonal count. Schlick's approximation is used in such cases to get better frame rates by significantly reducing the number of multiplications in the specular component. Since geometric moments are also based on monomials of the form $x^{p} y^{q}$, we could use Schlick's approximation to have a computationally simpler representation of the kernel, and a correspondingly fast algorithm to compute the invariants. In this paper we give the general formulation of normalised geometric moments and their invariant functions, and demonstrate how Schlick's approximation 
could be used effectively in this framework. Most importantly Schlick's approximation finds application in pattern recognition and image classification where normalized invariants are used as feature descriptors.

The organisation of this paper is as follows. The next section gives an overview of geometric moments and introduces normalized translation invariants. Schlick's approximating functions and their extensions that are suitable for defining normalized moments are given in Section 3. Experimental results showing the invariant characteristics of normalized moments using Schlick's approximation, as well as the reduction in computational complexity with the proposed method are provided in Section 4. Conclusions and possible extensions of the work presented in this paper are given in Section 5 .

\section{Geometric Moments and Invariants}

The two dimensional geometric moments of an image intensity distribution $I(x, y)$, is defined as follows [3]:

$$
m_{p q}=\int_{x=0}^{\infty} \int_{y=0}^{\infty} x^{p} y^{q} I(x, y) d x d y
$$

where $p, q$ are positive integers, and $m_{p q}$ denotes moments of order $p+q$. For an image of size $M \times N$ pixels $(0 \leq x<M, 0 \leq y<N)$, the above moment integral is often computed as

$$
m_{p q}=\sum_{x=0}^{M-1} \sum_{y=0}^{N-1} x^{p} y^{q} I(x, y)
$$

The coordinates of the image cetnroid can be computed from the first order moments as follows:

$$
\begin{aligned}
& \bar{x}=\frac{m_{10}}{m_{00}} \\
& \bar{y}=\frac{m_{01}}{m_{00}}
\end{aligned}
$$

The central moments defined below are used for translation-invariant recognition of images.

$$
\mu_{p q}=\sum_{x=0}^{M-1} \sum_{y=0}^{N-1}(x-\bar{x})^{p}(y-\bar{y})^{q} I(x, y)
$$


From the above equation, it is clear that central moments evaluated at high orders tend to become numerically unstable due to the monomial structure of the kernel functions. Image coordinate values are usually normalized to a value less than 1 by dividing by the image size, in order to eliminate this problem. With coordinate normalization, moment values tend to zero instead of infinity as the moment order increases. Normalization also helps in reducing the sensitivity of moment functions to image noise. We use the following definition for normalized translation-invariants:

$$
\hat{\mu}_{p q}=\sum_{x=0}^{M-1} \sum_{y=0}^{N-1}(\hat{x})^{p}(\hat{y})^{q} I(x, y)
$$

where

$$
\begin{aligned}
& \hat{x}=\frac{1}{M}(x-\bar{x}) \\
& \hat{y}=\frac{1}{N}(y-\bar{y})
\end{aligned}
$$

Both the above terms can take values in the range $[-1,1]$. The kernel of Eq.(5) involves product terms that can add to the complexity of a moment computation process, especially when the values of $p$ and $q$ are large. But this function can be easily approximated by a simple fraction containing two polynomials and only linear terms, at the same time preserving the desired invariant properties. This approximation can drastically reduce the amount of computations required for high order invariants.

\section{Schlick's Approximation}

The Schlick's approximation of an exponential function $x^{p}$, where $x \in[0,1)$, is given by [7]

$$
\begin{aligned}
S_{p}(x) & =\frac{x}{x+p-x p}, \quad p \in[1, \infty), \\
& =1, \quad \text { if } p=0 .
\end{aligned}
$$

The above formula is commonly used in Computer Graphics to approximate the term $(\cos \phi)^{f}$ that appears in the traditional Phong's illumination model for specular reflections [8], with a simple function that does not require eponentiation. Interestingly, $S_{p}(x)$ represents the ratio of a linear interpolation between 0 and 1 (given by $x$ in the numerator) to a linear interpolation between $p$ and 1 (given by $p(1-x)+x$ in the denominator), with $x$ as the parameter for interpolation. Thus $S_{p}(x)$ has a value ranging from 0 to 1 , for all $p$. Fig. 1 gives a plot of variations of $x^{p}$ with respect to $x \in$ $[0,1)$, for different values of $p$. Fig. 2 shows that the function in (7) closely approximates the values of $x^{p}$. It is also clear from the figure that Schlick's approximation provides a slower convergence to zero for $x<1$, as the value of $p$ is increased. 


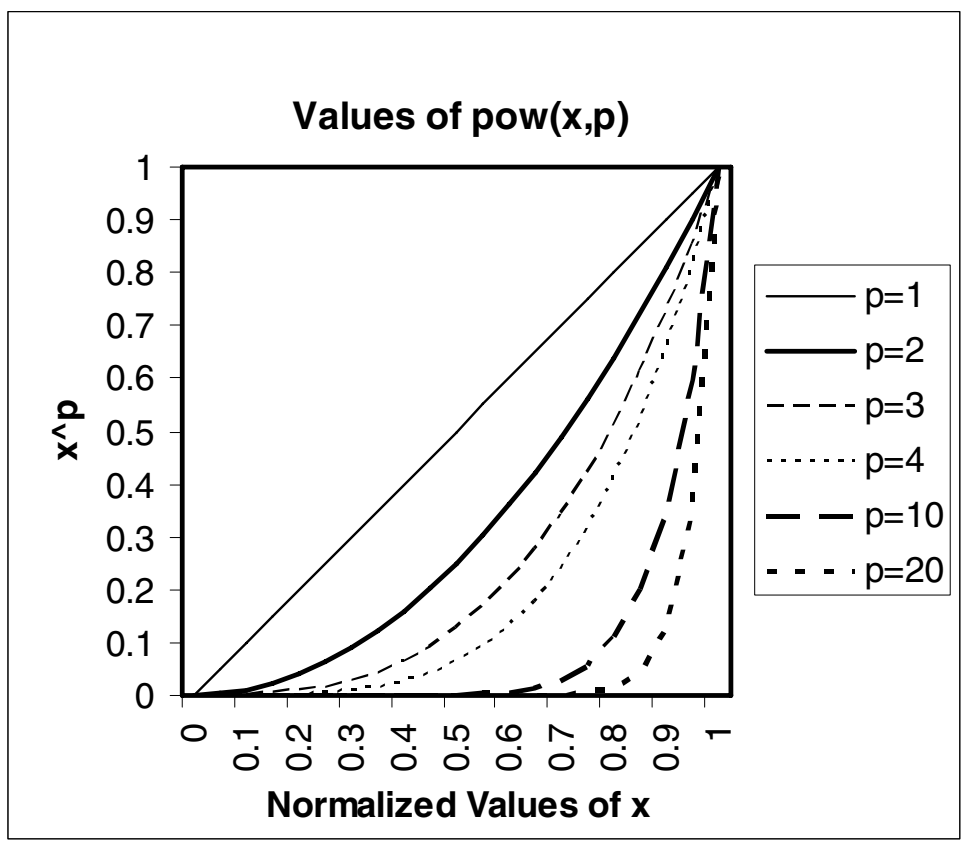

Fig. 1. A graph showing the values of the monomial $x^{p}$ for various values of $p$

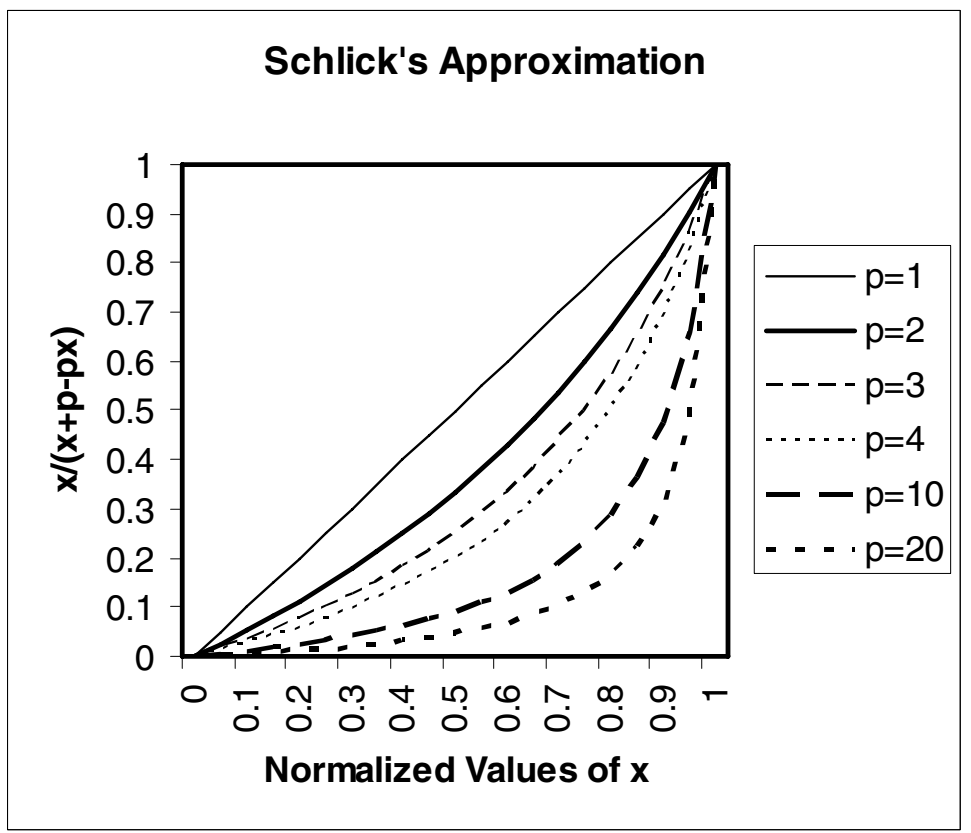

Fig. 2. A graph showing the approximations of $x^{p}$ using Schlick's functions, for different values of $p$ 
We note here that the normalized coordinate variables used in moment computation as given in Eq. (6) have an extended range $[-1,1]$. We correspondingly extend the Schlick's approximation function in Eq. (7), for $x \in(-1,1)$ as follows:

$$
\begin{array}{cl}
S_{p}(x)=\frac{x}{x+p-x p}, & x \geq 0, \quad p \in[1, \infty), \\
S_{p}(x)=\frac{(-1)^{p} x}{x-p-x p}, & x<0, \quad p \in[1, \infty), \\
S_{p}(x)=1, & \text { if } p=0 .
\end{array}
$$

The translation invariants given in Eq.(5) can now be rewritten based on the above definition as follows:

$$
\hat{\mu}_{p q}=\sum_{x=0}^{M-1} \sum_{y=0}^{N-1} S_{p}(x) S_{q}(y) I(x, y)
$$

In the following, we analyse the effect of approximation on accuracy and invariant characteristics of the above functions.

\section{Experimental Results}

In this section we present the results of a comparative analysis of the two methods for moment computation based on $\operatorname{Eqs}(5)$ and (9). Fig. 3 shows a binary image of size 200x200 pixels used in the experiments. For this image, the centroid (Eq.(3)) has coordinates $(106.4,105)$, with the origin of the image coordinate system located at the bottom-left corner.

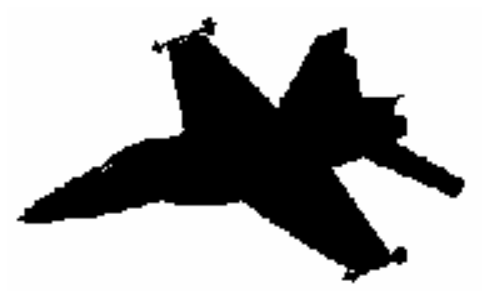

Fig. 3. Binary image of an aircraft model used for experimental evaluation of the proposed method

The values of $\hat{\mu}_{p 0}$ computed using Eqs.(5) and (9) for the above image are shown in Fig. 4. The values obtained using Schlick's approximation matches closely with the original values when $p$ is odd. Higher order moments tend to become zero because of coordinate normalization, but Schlick's approximation yields an offset when $p$ is even, and this offset could be useful in a feature vector used for pattern matching. 


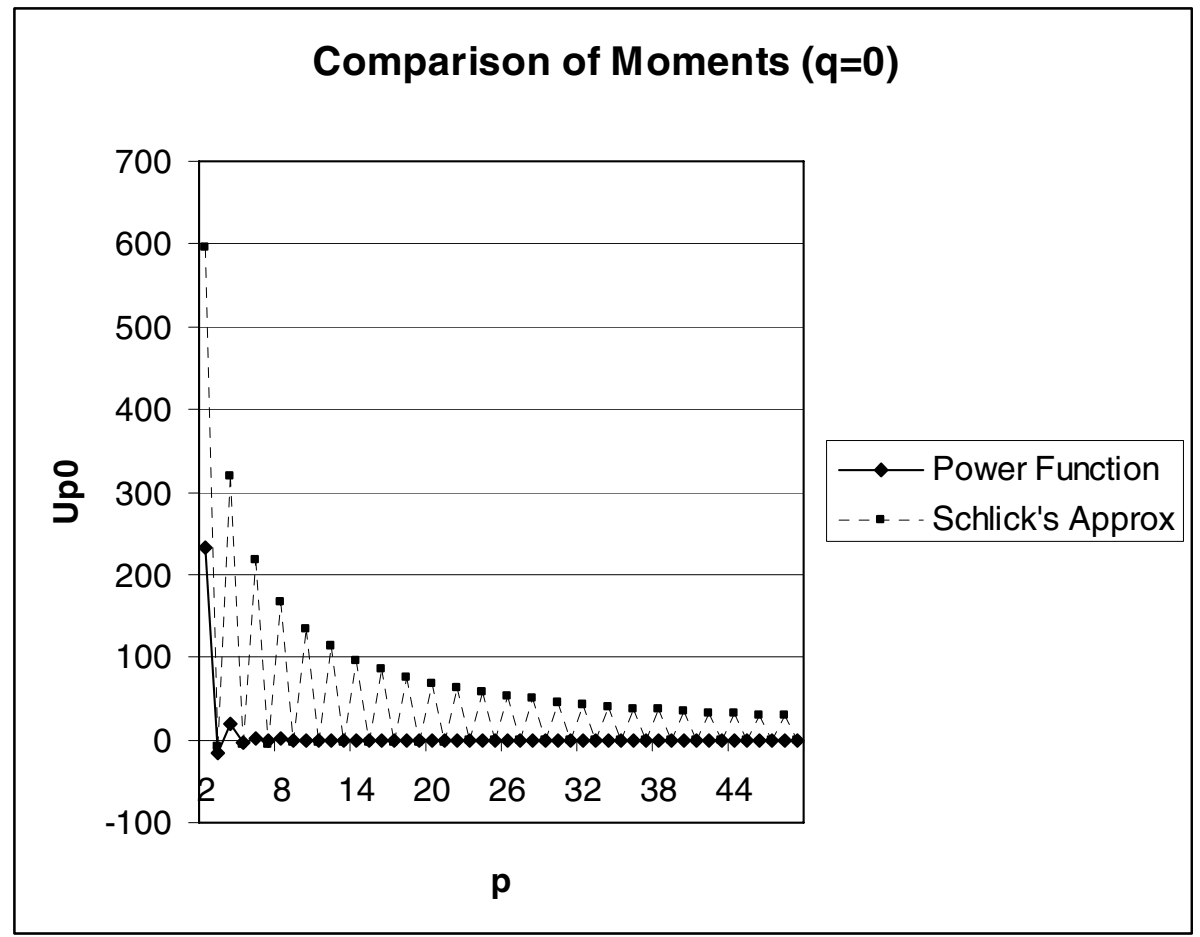

Fig. 4. Comparison of central moments with and without Schlick's approximation

This offset also appears for functions $\hat{\mu}_{p p}$, but converges to zero as the value of $p$ is increased.

The primary advantage a Schlick's approximation based moment function gives is the large reduction in the number of multiplications required for the monomial kernel. The computational time required for evaluating moments of order $p$ was analysed with the value of $p$ ranging from 0 to 100 (Fig. 5). The computations were performed on a $2.8 \mathrm{GHz}$ Intel-Pentium $4 \mathrm{CPU}$ with $1 \mathrm{~GB}$ of RAM. Figure 4 gives a comparison of CPU times with the values of $\hat{x}^{p}, \hat{y}^{q}$ in Eq. (5) computed using (i) repeated multiplication, (ii) built-in "pow()" function and (iii) Schlick's approximation (Eq. (8)). In all the three cases, moments were computed using the straight forward direct-sum method. The computational time could be further reduced by using the separabilityproperty of the kernel functions. This property is not affected by Schlick's approximation.

Moment functions that are invariant with respect to rotational transformations were derived by $\mathrm{Hu}$, using the theory of algebraic invariants. If we use the translation invariants given in Eq.(5) , (9) in Hu's algebraic expressions, then we get moments that are both translation and rotation invariant. Our experiments show that moments 


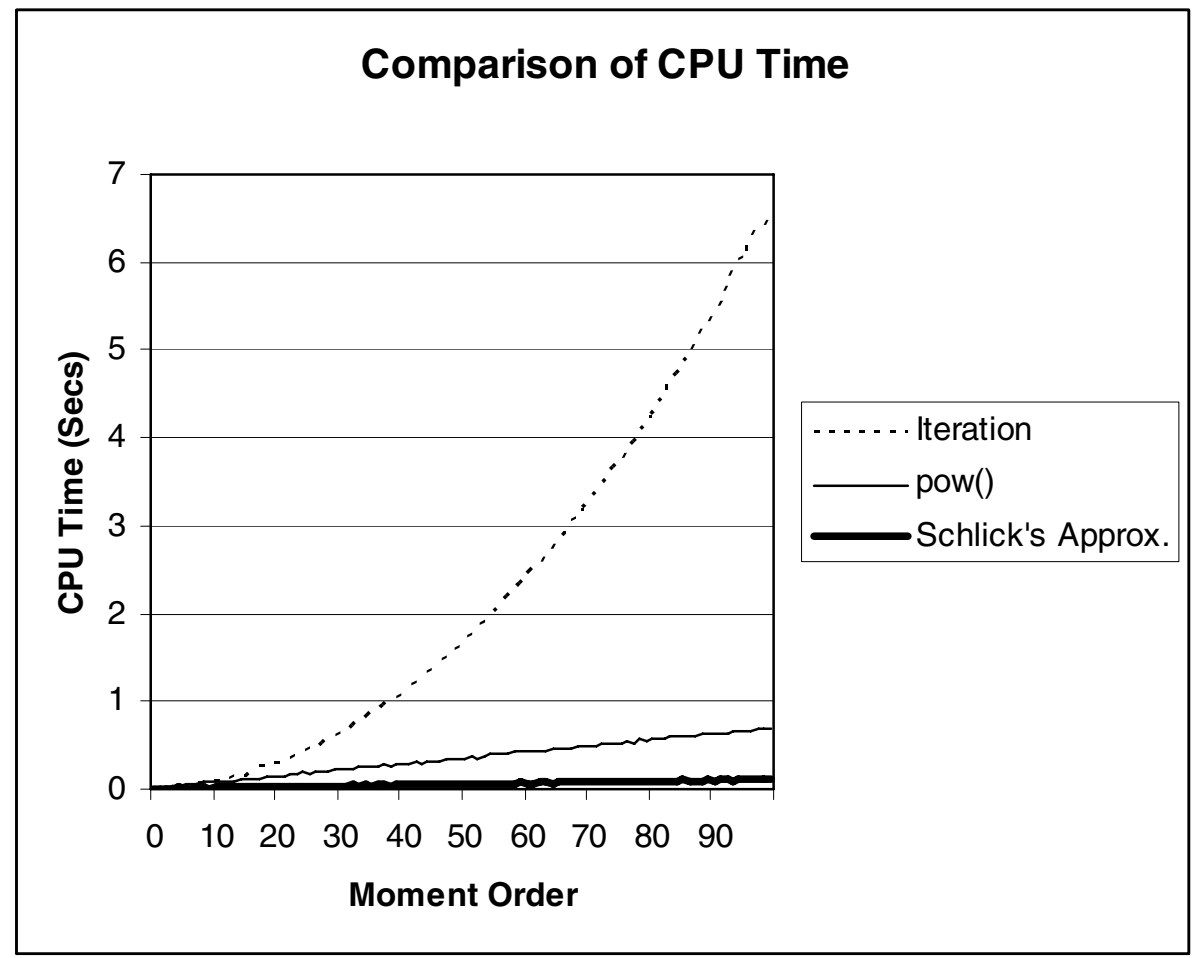

Fig. 5. Comparison of CPU times with the moment kernel computed using the exponential function and Schlick's approximation

obtained using Schlick's approximation can be utilized to form invariants, but we have to take note of the errors introduced by the approximation. There is clearly a trade-off between the approximation errors and the computational advantage that Schlick's approximation provides in the calculation of invariants. As an example, we consider a fourth-order invariant given by

$$
K_{4}=\hat{\mu}_{40}+2 \hat{\mu}_{22}+\hat{\mu}_{04}
$$

The plots in Fig. 6 can be used to verify the invariant characteristics of the above function, computed with and without Schlick's approximation. It may also be noted that the error introduced by Schlick's approximation reduces with order (Fig. 4), and higher order invariants tend to become more accurate when compared with invariants formed with monomial functions.

The translation invariant characteristics are not found to be affected by Schlick's approximation. This is because the parameter in Schlick's function is computed after translating the image to the centroid, as given in Eq. (6). 

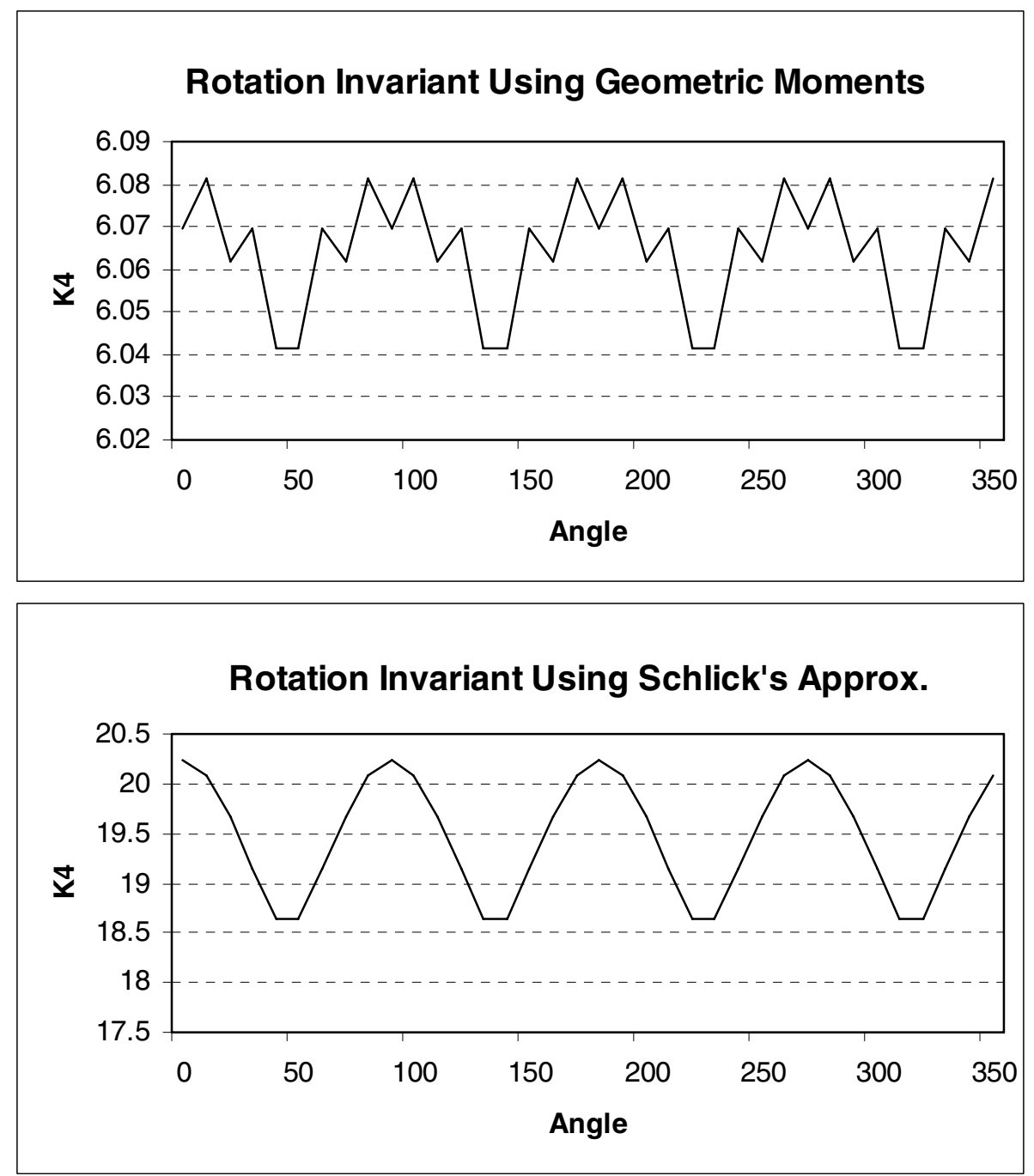

Fig. 6. Comparison of moment invariants computed using original moments and Schlick's approximation

\section{Conclusion}

This paper has shown that Schlick's approximation for the function $x^{p}$ could be used to compute normalized geometric moments and invariants for applications in pattern recognition. The approximation replaces the power functions in the kernel of the moment definition by simple rational polynomials, resulting in a significant reduction of the amount of computations. The paper has used an extended version of Schlick's approximation to suit the range $[-1,+1]$ of the normalized coordinates. Experimental 
results have shown that while translation invariant characteristics can be accurately preserved in central moments computed using Schlick's approximation, rotation invaraints have larger error introduced range and magnitude. This error, however is found to be within acceptable range for image classification and recognition. The computational advantage provided by Schlicks approximation far outweighs the problems associated with an increase in the variance of moment values.

The paper has thus introduced a mathematical tool used in the field of Computer Graphics to the image analysis area. Schlick's approximation could be used in conjunction with other methods for fast computation of moments. Further work in this area could be directed towards an analysis of approximation errors in the presence of image noise, and the derivation of generalized affine invariants using Schlick's approximation.

\section{References}

1. Ghorbel, F., Derrode, S., Dhahbi, S.: Reconstructing with Geometric Moments. In: Proc. Int. Conf. on Machine Intelligence: ACIDCA-ICMI 2005 (2005)

2. Li, B.: High-Order Moment Computation of Gray Level Images. IEEE Trans. On Image Processing 4, 502-505 (1995)

3. Mukundan, R., Ramakrishnan, K.R.: Moment Functions in Image Analysis - Theory and Applications. World Scientific, Singapore (1998)

4. Yang, L.R., Albregtsen, F.: Fast and Exact Computation of Cartesian Geometric Moments Using Discrete Greens Theorem. Pattern Recognition 29, 1061-1073 (1996)

5. Martinez, J., Thomas, F.: Efficient Computation of Local Geometric Moments. IEEE Trans. On Image Processing 11, 1102-1111 (2002)

6. Liu, W., Chen, S.S., Cavin, R.: A Bit-Serial VLSI Architecture for Generating Moments in Real Time. IEEE Trans. On. Systems Man and Cybernetics 23, 539-546 (1993)

7. Schlick, C.: A Fast Alternative to Phong's Specular Model. In: Heckbert, P.S. (ed.) Graphics Gems IV, pp. 385-387. Elsevier Science, Amsterdam (1994)

8. Shirley, P., Smits, B., Hu, H., Lafortune, E.: A Practioner's Assessment of Light Reflection Models. In: Proc. 5th Pacific Conf. on Computer Graphics and Applications, pp. 40-49 (1997) 